\title{
Knockdown of FUT3 disrupts the proliferation, migration, tumorigenesis and TGF- $\beta$ induced EMT in pancreatic cancer cells
}

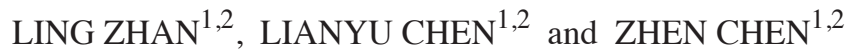 \\ ${ }^{1}$ Department of Integrative Oncology, Fudan University Shanghai Cancer Center; \\ ${ }^{2}$ Department of Oncology, Shanghai Medical College, Fudan University, Shanghai 200032, P.R. China
}

Received June 27, 2016; Accepted March 7, 2018

DOI: $10.3892 / \mathrm{ol} .2018 .8738$

\begin{abstract}
Fucosyltransferases (FUTs) are critical for glycoproteins and glycolipid chains and serve an important role in the adhesive interaction between selectins and their ligands, which contribute to tumor cell spread and metastasis. While multiple cancer cell lines heavily express FUT3, the present study investigated the expression level of FUT3 in different human pancreatic cancer cell lines. Forced expression and knockdown of FUT3 in different pancreatic cancer cell line demonstrated that FUT3 is important in cell proliferation. Using wound healing and transwell assays, it was observed that the migratory ability was decreased in FUT3 downregulated Capan-1 cell line, compared with the normal Capan-1 cell line. Furthermore, it was demonstrated that the knockdown of FUT3 impaired the adhesion of Capan-1 with E-selectin and inhibited transforming growth factor (TGF)- $\beta$-induced epithelial-mesenchymal transition. These data suggest that the knockdown of FUT3 inhibits the tumorigenesis in vivo and FUT3 may be a promising target aiming at reducing the metastatic virulence of pancreatic cancer cells.
\end{abstract}

\section{Introduction}

Pancreatic cancer is one of the most threatening malignancies characterized by aggressive growth and a high metastatic ability during the early stage. The 5-year survival rate is only $6 \%$ (1), and only $10-20 \%$ of patients with pancreatic cancer are eligible for surgery at the time of diagnosis (2). The mechanism of tumor cell invasion and metastasis remains unclear (3). Therefore, an improved understanding of the metastatic process in pancreatic cancer may assist in developing certain effective therapies.

Correspondence to: Professor Zhen Chen, Department of Integrative Oncology, Fudan University Shanghai Cancer Center, 270 Dong An Road, Shanghai 200032, P.R. China

E-mail: cz.chenzhen@gmail.com

Key words: fucosyltransferase, metastasis. E-selectin, epithelial-to-mesenchymal transition, pancreatic cancer
A number of studies have suggested the involvement of sialyltransferase and fucosyltransferases (FUTs) in pancreatic cancer progression. It has been demonstrated that pancreatic cancer metastasis and invasion are mediated by modification of the cell surface with attachment of glycosyl residues by these enzymes $(4,5)$.

Sialyl-Lewis A (SLea) or sialyl-Lewis X (SLex), the E-selectin ligand glycans, are extensively expressed on numerous types of cancer cell surface, including colorectal, pancreatic, gastric, breast, prostate and lung cancer $(6,7)$. It has been previously confirmed that SLea and SLex contribute to the metastatic process, thereby constituting the initial step in tumor cell extravasation (6).

FUTs belong to the Golgi apparatus enzyme family that transfer L-fucose sugars from GDP fucose donor substrates to a glycoside or a peptide (8). FUTs are subdivided into FUT1 and FUT2 ( $\alpha-1,2)$, FUT3, FUT4, FUT5, FUT6, FUT7, FUT8 $(\alpha-1,6)$ and FUT9 $(\alpha-1,3 / 4)$, according to the fucosylation site $(9,10)$. FUT3 is an $\alpha-1,3 / 4$ fucosyltransferase that is absorbed by red blood cells and results in the Lewis phenotype; it generally transfers fucose residue to type I disaccharides compared with type II disaccharides (11).

The role of sialyltransferase and FUTs in cancer metastasis has been improved by numerous previous studies: It was observed that elevated sialyltransferase levels or FUTs expression indicated high metastatic potentiality and poor prognosis in epithelial cancers (12-15). Knockdown of the expression of FUT significantly reduced metastatic capacity of human pancreatic and colon cancer cells (16-18).

It has been demonstrated that Epithelial-mesenchymal transition (EMT) serves a crucial role in the early phase of cancer metastasis $(19,20)$. EMT is characterized by a phenotype transition from epithelial to mesenchymal, featured by the transformation of an epithelial shape into a fibroblast-like morphology (21). The process is accompanied by a series of enhanced activity of transcriptional repressors including Snail Family Transcriptional Repressor 1 (SNAIL1), Zinc Finger E-box Binding Homeobox 1 and Twist Family BHLH Transcription Factor 1 that downregulate the expression level of epithelial (E)-cadherin $(7,22,23)$. A previous study indicated that FUTs were associated with EMT in colorectal cancer $(24,25)$.

In the present study, FUT3 expression levels in a pancreatic ductal epithelial cell line and 4 different pancreatic cancer 
cell lines were analyzed. The involvement of FUT3 in the cell proliferation rate, mobility and potential to form tumors in an animal model was also investigated. In addition, the association between FUT3 and EMT in cancer metastasis was assessed. The present study demonstrated that FUT3 affected pancreatic cancer cell proliferation and migration, and that downregulation of FUT3 inhibits TGF- $\beta$-induced EMT and disrupts the tumor formation in an animal model.

\section{Materials and methods}

Cell lines and mice. The human pancreatic ductal epithelial HPDE6-C7 cell line was obtained from Hongshun Biotech Corporation (Shanghai, China). The human pancreatic cancer BxPC-3, Capan-1, Mia PaCa-2 and SW-1990 cell lines were obtained from the American Type Culture Collection (Manassas, VA, USA). The 293T cell line for transfection was purchased from The Cell Bank of Type Culture Collection of Chinese Academy of Sciences (Shanghai, China). All cells were cultured in Dulbecco's modified Eagle medium (DMEM; Gibco; Thermo Fisher Scientific, Inc., Waltham, MA, USA) supplemented with $10 \%$ fetal bovine serum (FBS; Gibco; Thermo Fisher Scientific, Inc.) at $37^{\circ} \mathrm{C}$ with $5 \% \mathrm{CO}_{2}$. A total of 20 female BALB/c-nu/nu nude mice aged 4-6 weeks were obtained from the Shanghai Institute of Materia Medica, Chinese Academy of Sciences (Shanghai, China), housed at $24^{\circ} \mathrm{C}$ and $65 \%$ humidity, with an alternating $12: 12 \mathrm{~h}$ light/dark cycle and allowed free access to food and tap water in accordance with the Guide for the Care and Use of Laboratory Animals (26).

Antibody and reagents. The anti-FUT3 (cat. no., ab110082) antibody was purchased from Abcam (Cambridge, UK), and anti-E-cadherin (cat. no., A3044), anti-Vimentin (cat. no., A1504) and anti-SNAIL1 (cat. no., A5243) antibodies were purchased from ABclonal Biotech Co., Ltd. (Woburn, MA, USA), anti- $\beta$-actin (cat. no., 4967S, 1:2,000) was purchased from Univ-Bio (Shanghai, China), goat anti-mouse/rabbit secondary antibody were purchased from Thermo Fisher Scientific, Inc. (cat. no., 31430/31460) Recombinant human transforming growth factor $\beta$ (TGF- $\beta 1$ ) was obtained from PeproTech, Inc. (cat. no., 100-21C, Rocky Hill, NJ, USA).

Plasmids and transfection. For the induced FUT3 expression, a cDNA expression vector (2 $\mu \mathrm{g}$, cat. no., RC211069) was purchased from OriGene Technologies, Inc. (Rockville, MD, USA) and introduced into Mia PaCa-2 cells using LipoFiter reagent (cat. no., HB-TRLF-1000; Hanbio Biotechnology Co., Ltd., Shanghai, China). Short hairpin (sh)RNAs against human FUT3 were generated by Dharmacon GIPZ ${ }^{\mathrm{TM}}$ plasmids (GE Healthcare Dharmacon, Inc., Lafayette, CO, USA). A total of 2 different sequences of shRNA for the FUT3 gene were examined (Clone ID: V2LHS_83410, 5'-TATAAGTGG TGGTCCTGGG-3' and V3LHS_392930, 5'-CCAAGTTGA ACCAGATCCA-3' $10 \mu \mathrm{g}$ lyophilized plasmid for each). To produce FUT3 knockdown cell line, lentiviruses were generated by transient transfection into $293 \mathrm{~T}$ cells as previously described (27). Briefly, $10 \mu \mathrm{g}$ of shRNA plasmid and $10-\mu \mathrm{g}$ package vector were transfected into $10 \mathrm{~cm}$ diameter dishes plated with $293 \mathrm{~T}$ cells $(70-80 \%$ confluence). The medium
(10 ml) was collected following a $48 \mathrm{~h}$ incubation at $37^{\circ} \mathrm{C}$ and filtered through $0.22 \mu \mathrm{m}$ cellulose acetate filters. Capan-1 cells were cultured in the previous collected lentivirus media at $37^{\circ} \mathrm{C}$ for 2 days, and stable cell lines expressing the FUT3 shRNA were selected with puromycin at $5 \mu \mathrm{g} / \mathrm{ml}$.

Proliferation assay. In 96-well plates, 5x10 3 Mia PaCa-2 and Capan-1 cells were added to each well and incubated in a humidified $5 \% \mathrm{CO}_{2}$ atmosphere at $37^{\circ} \mathrm{C}$. After $0,1,2,3,4$ and 5 days, cells were washed by PBS for three times and cell proliferation was measured daily using a Cell Counting Kit-8 according to the manufacturer's protocols (CCK-8; cat. no., CK04, Dojindo Molecular Technologies, Inc., Rockville, MD, USA). The growth curve was drawn according to the optical densityvalues obtained from CCK-8 assay. Plates were read using spectrophotometric analysis at a wavelength of $450 \mathrm{~nm}$ using the SpectraMax M5e microplate reader (Molecular Devices, LLC, Sunnyvale, CA, USA) and SoftMax Pro version 6.4 software (Molecular Devices). The experiments were performed in triplicate, and at least three independent assays were performed.

Wound healing assay. A total of $5 \times 10^{5}$ Capan-1 cells were seeded in 6-well plates. Once the monolayer had reached $70-80 \%$ confluence, a 500-600- $\mu \mathrm{m}$ diameter scratch was created longitudinally using a sterile pipette tip. Following scratching, the wells were gently washed twice with fresh medium to remove the detached cells. Images of the monolayer were captured at $0,1,2$ and 3 days using a Leica DMIRB light microscope (x200 magnification). A total of 4 different views of the wound were marked, and the distance was quantitatively evaluated using Leica IM50 Image Manager version 4.0 software (Leica Camera AG, Solms, Germany).

Transwell migration assay. Cell migration was evaluated using modified $8 \mu \mathrm{m}$ Boyden chambers (Corning Incorporated, Corning, NY, USA). A total of 3x10\%/well Capan-1 cells were seeded onto upper chamber containing $400 \mu 1$ DMEM with 1\% FBS and 2 ml DMEM (Gibco; Thermo Fisher Scientific, Inc.) was added to lower chamber. Following incubation in a humidified $5 \% \mathrm{CO}_{2}$ atmosphere at $37^{\circ} \mathrm{C}$ for $18 \mathrm{~h}$, the non-migrated cells that remained on the upper side of the filter membrane were gently removed with a cotton swab. The cells on the lower side of the insert filter were fixed by $95 \%$ ethanol at room temperature for $15 \mathrm{~min}$ and stained with $200 \mu \mathrm{l}$ hematoxylin $(0.5 \%, 5 \mathrm{~min})$ and $200 \mu \mathrm{l}$ eosin $(1 \%, 1 \mathrm{~min})$ at room temperature (cat. no., C0105, Beyotime, Shanghai, China) according to the manufacturer's protocol. The number of cells on the lower side of the filter was counted under light field of Leica DMIRB microscope (x200) in 10 random fields. The average number of migrated cells per well was recorded from 3 separate experiments.

E-selectin binding assay. Rh-E-selectin ( $5 \mathrm{~g} / \mathrm{ml}$; R \& D Systems, Inc., Minneapolis, MN, USA) or $1 \%$ bovine serum albumin (cat. no., A8020; Beijing Solarbio Science \& Technology Co., Ltd., Beijing, China) was coated onto 96 -well microplates at $4^{\circ} \mathrm{C}$ for $24 \mathrm{~h}$. Following washing 3 times with assay buffer $(20 \mathrm{mM}$ Hepes, pH 7.4 with $150 \mathrm{mM} \mathrm{NaCl}$ and $1 \mathrm{mM} \mathrm{CaCl}_{2}$ ), $1 \times 10^{5}$ Capan-1:shNT (shRNA non-targeting) and Capan-1:shFUT3 
A

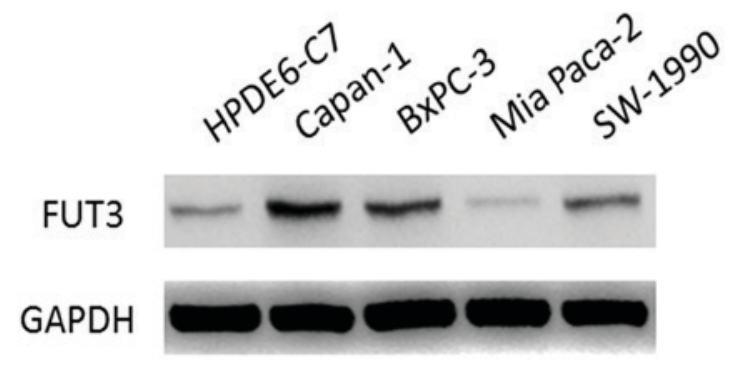

B

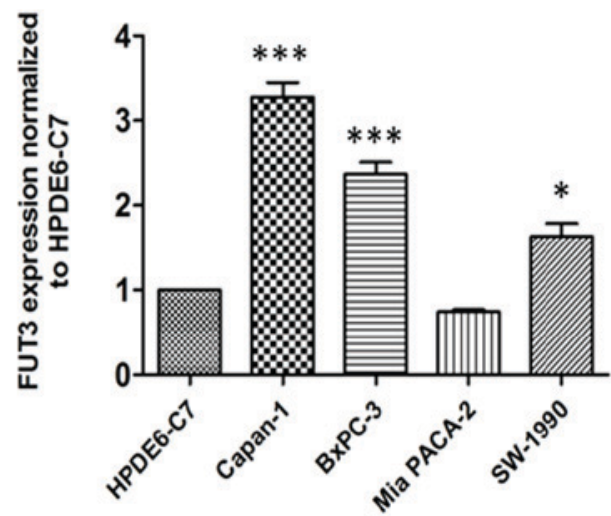

Figure 1. Differential expression of FUT3 between pancreatic ductal epithelial cell line and pancreatic cancer cell lines. (A) Expression level of FUT3 in different cell lines. (B) Quantification of FUT3 expression normalized to HPDE6-C7. FUT3, fucosyltransferases 3 . ${ }^{*} \mathrm{P}<0.05$ and ${ }^{* * *} \mathrm{P}<0.001$ in comparison with HPDE6-C7.

cells were added. Followed by incubation at $37^{\circ} \mathrm{C}$ for $1 \mathrm{~h}$, adherent cells were examined with $50 \mu \mathrm{l}(5 \mathrm{mg} / \mathrm{ml})$ Thiazolyl Blue (Sigma-Aldrich; Merck KGaA, Darmstadt, Germany) based on a colorimetric method. Plates were read using spectrophotometric analysis at a wavelength of $570 \mathrm{~nm}$ using the SpectraMax M5e microplate reader (Molecular Devices, LLC, Sunnyvale, CA, USA) and SoftMax Pro version 6.4 software (Molecular Devices). All the experiments were repeated in quintuplicate, and three independent assays were undertaken. Numbers of adhered cells are presented as mean \pm standard deviation (SD).

Western blot analysis. Total protein was extracted with radioimmunoprecipitation assay lysis buffer (cat. no., 89900, Thermo Scientific) with protease and phosphatase inhibitors (Roche Applied Science, Penzberg, Germany). Total proteins were quantified using a BCA kit (Pierce; Thermo Fisher Scientific, Inc.; cat. no., 23225) and subjected to $12 \%$ SDS/PAGE gel with $50 \mu \mathrm{g}$ in each lane, followed by transfer onto polyvinylidene fluoride membranes (Bio-Rad Laboratories, Inc., Hercules, CA, USA). Membranes were blocked with 5\% nonfat milk in TBST (100 mmol/1 Tris-HCl, 1\% Tween 20, $\mathrm{pH} 7.5$ ) for $1 \mathrm{~h}$ at room temperature, and then incubated with appropriate aforementioned primary antibodies in 1:2,000 dilution at $4^{\circ} \mathrm{C}$ overnight. Signals were detected using the aforementioned horseradish peroxidase-conjugated secondary antibody in 1:5,000 dilution (Thermo Scientific) and an electrochemiluminescence kit (cat. no., RPN2109; Amersham; GE Healthcare Life Sciences, Uppsala, Sweden). The quantitative analysis was performed using Tanon image version 1.0 software (Tanon Science and Technology, Co., Ltd., Shanghai, China).

Xenograft tumor models. All studies involving animal manipulations were approved by the Fudan University Shanghai Medical College Animal Care and Use Committee and followed the National Institutes of Health guidelines for the care and use of animals (approval no., 20150330A035). FUT3-knockdown or wild type Capan 1 cells $\left(2 \times 10^{6}\right.$ in $0.2 \mathrm{ml}$ $\mathrm{DMEM} /$ mouse) were injected subcutaneously into the right axilla of randomized groups of female nude mice (shFUT3-2 and shNT group, 10/group). The tumor growth was measured weekly using calipers by the formula $\left(\mathrm{a} \mathrm{x}^{2}\right) \times 0.5$, where $a$ and $b$ represent the height and width, respectively. All mice were sacrificed on day 21 . The tumors were removed and weighed. Experiments were performed with 6 mice per group.

Statistical analysis. One-way ANOVA was used in statistics involving cell migration and proliferation. Un-paired Student's t-test was used for statistics involving tumor volume. $\mathrm{P}<0.05$ was considered to indicate a statistically significant difference. All group results are presented as mean \pm standard deviation (SD). GraphPad Prism 5 (GraphPad Software, Inc., La Jolla, CA, USA) was used for these analyses.

\section{Results}

FUT3 exhibits high expression levels in pancreatic cancer cell lines. FUT3 was highly expressed in all pancreatic cancer cell lines but was poorly expressed in the normal pancreatic ductal epithelial HPDE6-C7 cell line and Mia PaCa-2 cell line (Fig. 1A). The expression level of FUT3 in Capan-1 was significantly increased 3.5-fold compared with HPDE6-C7 $(\mathrm{P}<0.001)$, while in Mia $\mathrm{PaCa}-2$ cells it was not significantly increased 0.7-fold compared with HPDE6-C7 $(\mathrm{P}=0.25$; Fig. 1B).

FUT3 affects proliferation and cell migration in pancreatic cancer cells. To explore the functional importance of FUT3, FUT3 overexpression plasmid was transfected into Mia PaCa-2 and FUT3 shRNA plasmid into Capan-1 cells. As indicated in Fig. 2A and B, overexpressed FUT3 led to a significant increase in Mia $\mathrm{PaCa}-2$ cell proliferation $(\mathrm{P}<0.05)$, The 2 shRNA reduced FUT3 protein to markedly low levels in Capan-1 cells compared with shNT cells (Fig. 2C). Knockdown of FUT3 in Capan-1 significantly inhibited the cell proliferation rate $(\mathrm{P}<0.01$; Fig. 2D). A wound healing assay was then performed to evaluate the effect of FUT3 on the migratory capacity of Capan-1 cells. A reduction in the migratory ability of the Capan-1:shFUT3 cell line was detected at $3 \mathrm{~d}$ in comparison with shNT control cell line (Fig. 3A and B). The transwell assay indicated that the number of migrated FUT3 knockdown Capan-1 cells (shFUT3-2) was decreased 
A

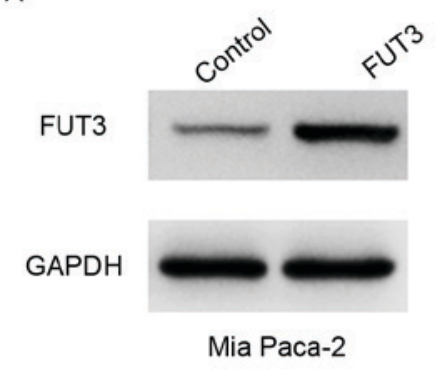

C

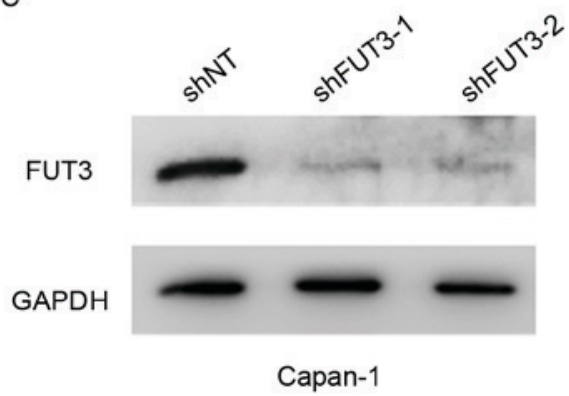

B

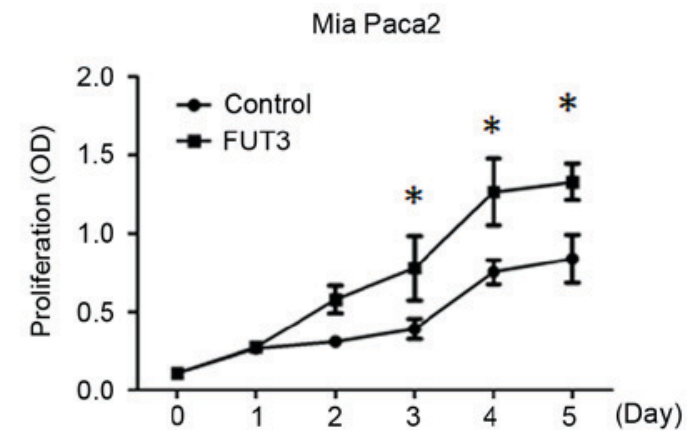

D

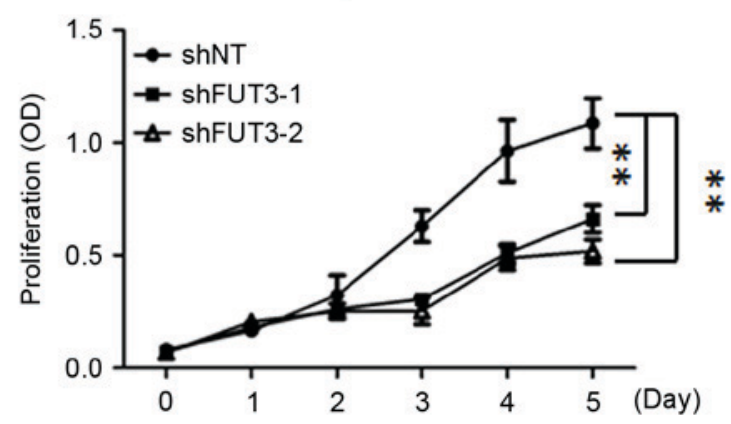

Figure 2. Expression of FUT3 associated with pancreatic cancer cell proliferation. (A) Forced expression of FUT3 in Mia PaCa-2 demonstrated by western blotting. (B) Forced expression of FUT3 promoted the Mia PaCa-2 cell proliferation rate. (C) The knockdown of FUT3 in Capan-1 demonstrated by western blotting. (D) The knockdown of FUT3 inhibited the Capan-1 cell proliferation rate. ${ }^{*} \mathrm{P}<0.05$ and ${ }^{* *} \mathrm{P}<0.01$, compared with the control or shNT. FUT3, fucosyltransferases 3; OD, optical density; shNT, short hairpin nontargeting.

A
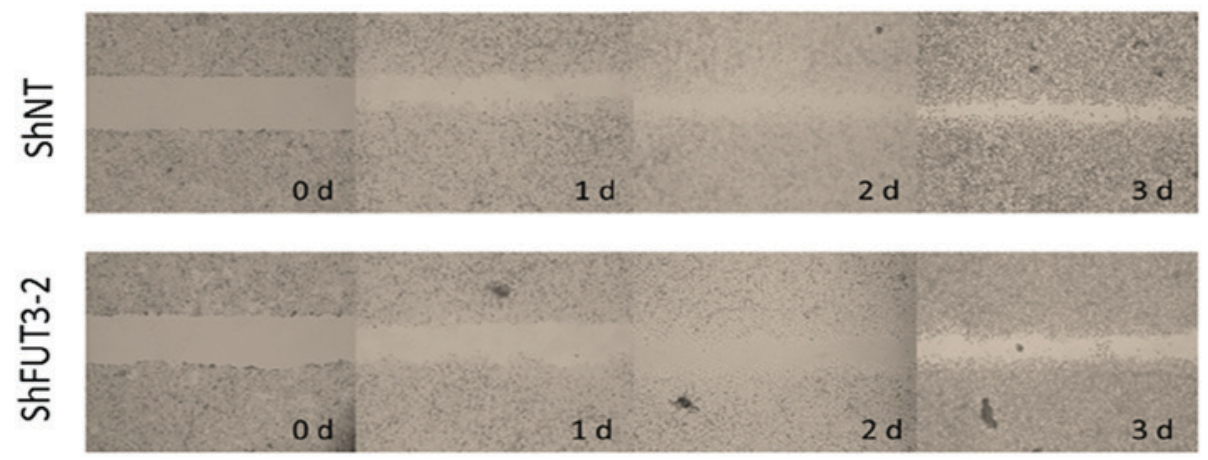

B

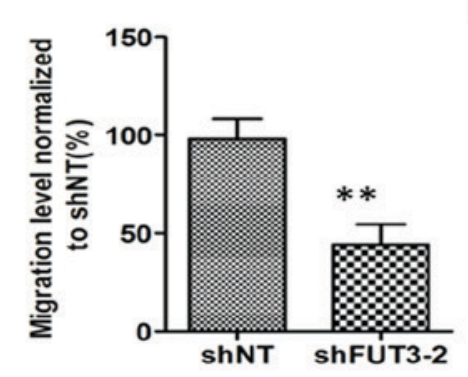

C

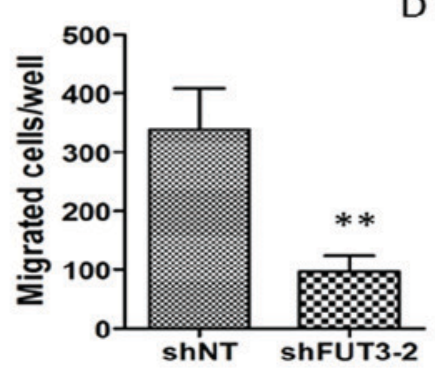

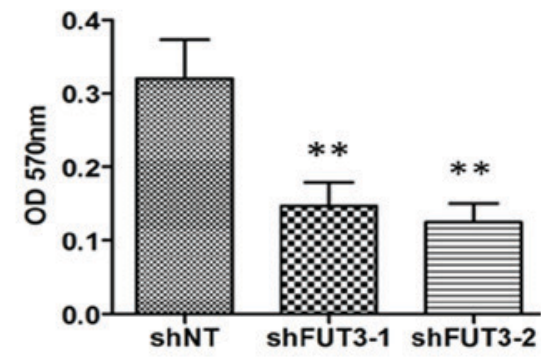

Figure 3. Cell migration and adhesion analysis of Capan-1 shRNA cells. (A) Wound image at 0,1,2 and 3 days. (B) Quantification of shFUT3 cell migration expressed as percentage of migration with shNT. (C) Migrated cells per well following $18 \mathrm{~h}$ incubation. (D) Binding of Capan-1 shRNA cells to E-Selectin. Values are expressed as mean \pm standard deviation. ${ }^{* *} \mathrm{P}<0.01$ vs. shNT group. FUT3, fucosyltransferases 3; OD, optical density; shNT, short hairpin nontargeting.

almost 3-fold, compared with the shNT (Fig. 3C). E-selectin binding assays were performed to determine whether FUT3 affected the adhesion of Capan-1. In comparison with the
Capan-1 cells, a significant decrease in the level of adhesion of Capan-1:shFUT3-1/2 cells to E-selectin was indicated (Fig. 3D). 
A

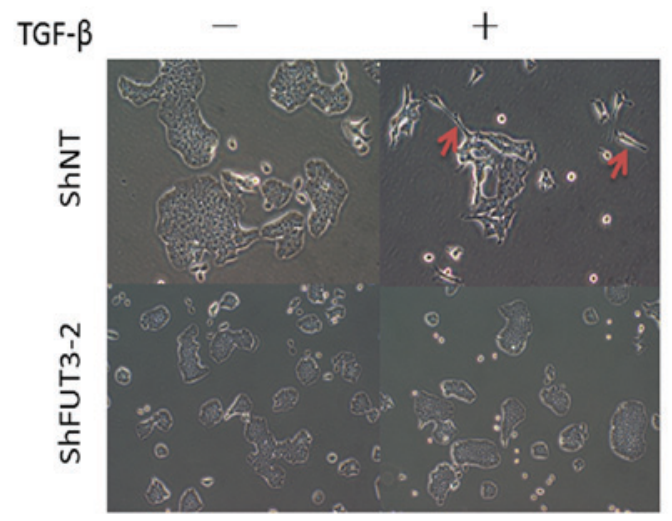

B

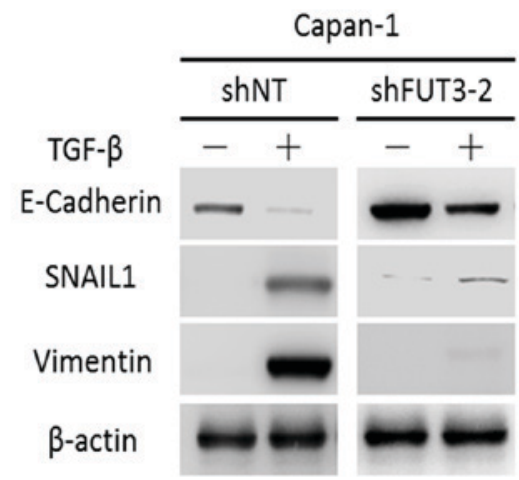

Figure 4. Induction of EMT in pancreatic cancer cell by TGF- $\beta$ is inhibited by knockdown of FUT3. (A) Cells treated with TGF- $\beta$ exhibiting fibroblastic morphology (x400 magnification, red arrows). (B) Expression of marker genes for EMT were determined by western blot. FUT3, fucosyltransferases 3 ; TGF- $\beta$, transforming growth factor- $\beta$; EMT, epithelial-mesenchymal transition; SNAIL1, Snail Family Transcriptional Repressor 1; E-cadherin, epithelial cadherin.

A

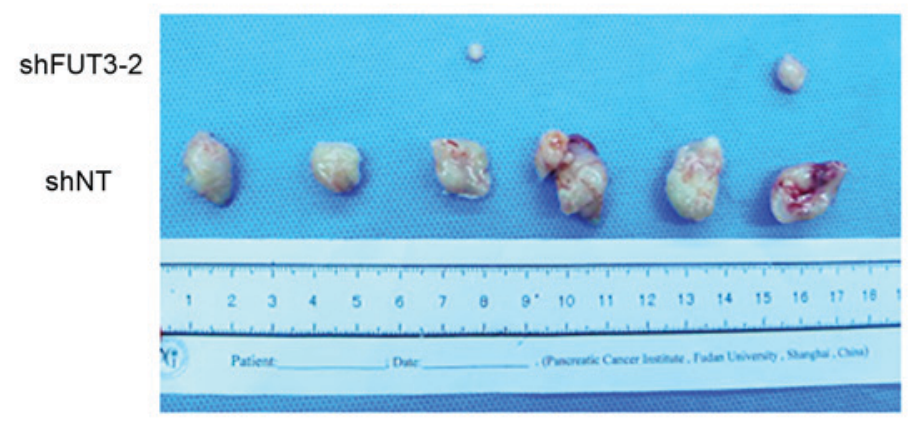

B

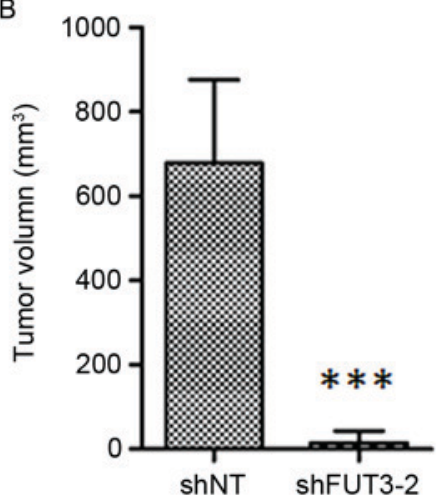

Figure 5. Knockdown of FUT3 inhibits tumor growth in vivo. (A) Images of tumors arising from subcutaneously transplanted FUT3-knockdown and negative control cells. (B) The mean tumor volumes of each group were measured. The mean \pm standard deviation was determined in each group. ${ }^{* * *} \mathrm{P}<0.001 \mathrm{vs}$. shNT group. FUT3, fucosyltransferases 3; shNT, short hairpin nontargeting.

Knockdown of FUT3 in Capan-1 inhibits TGF- $\beta$-induced EMT. To detect Capan-1 cells undergoing EMT, Capan-1 introduced with shNT or shFUT-2 cell lines were maintained in DMEM supplemented with $10 \% \mathrm{FBS}$ or in serum-free medium supplemented with TGF- $\beta$ (50 ng/ml). Following incubation for $7 \mathrm{~d}$, cells were observed with a phase-contrast microscope. Treatment with TGF- $\beta$ induced a fibroblast-like appearance in Capan-1 shNT cells, but not in Capan-1:shFUT3 cells (Fig. 4A). TGF- $\beta$ treatment increased the expression of mesenchymal marker genes SNAIL1 and vimentin, whereas the level of E-cadherin was downregulated in the shNT cell line. However, in the FUT3 knockdown cell line, the change was limited by visual observation (Fig. 4B). These results indicated that the downregulation of FUT3 inhibits TGF- $\beta$-induced EMT in Capan-1 cells.

Effect of FUT3 on the Capan-1 cell tumorigenicity in vivo. Marked tumorigenicity was observed in the shNT group 21 days following subcutaneous inoculation, but it was almost impossible to initiate tumorigenesis in the shFUT3 group (Fig. 5A). The average sizes of tumors were $678.83 \pm 88.2$ and $14.83 \pm 12.62 \mathrm{~mm}^{3}$ for the shNT and shFUT3 groups, respectively, which was significantly different $(\mathrm{P}<0.001)$. These results indicate that FUT3 altered the tumorigenicity capacities of Capan-1 cells in vivo (Fig. 5B)

\section{Discussion}

Increased glycosyltransferases expression has been demonstrated in pancreatic adenocarcinoma tissues $(28,29)$. In addition, immunohistochemistry data indicated an association between tumor differentiation and overexpression of the N-acetylgalactos aminyltransferases GalNAcT3 and GalNAc-T6 in pancreatic adenocarcinoma $(30,31)$. Certain studies have indicated that pancreatic tumor cells presented an enhanced expression of SLea and SLex antigens, which serve an essential role in adhesion to epithelium and the formation of metastasis $(32,33)$. Other studies have suggested that enhanced FUT3 activity induces SLea and SLex expression, and potentiates the malignant potential of metastatic cancer cells (6). The results of the present study have demonstrated that FUT3 was highly expressed in pancreatic carcinoma cell lines compared with a normal pancreatic ductal epithelial cell line. The functional effects of FUT3 were assessed in cell proliferation, transwell and wound healing assays in pancreatic cancer cells, and it was identified that the forced expression of FUT3 
increased the proliferation rate of pancreatic cancer cells, and its knockdown suggested a reverse in proliferation ability.

In cancer cells, certain studies have identified that Lewis antigens exhibited an alteration in expression pattern and that SLex demonstrated a correlation with poor prognosis in gastric cancer $(34,35)$. It has been revealed that the combination of SLex and E-Selectin molecules facilitates tumor cell extravasation, and SLex is also correlated with an invasive phenotype of tumor cells $(36,37)$. The present study validated that the knockdown of FUT3 in Capan-1 cells decreased the adhesion of cancer cells to E-selectin, which is closely associated with the process of metastasis. These data were in accordance with previous studies (38).

It has been suggested that glycans exhibit a marked correlation with EMT (24). Glycosphingolipids have a significantly downregulated expression level during the EMT process, either induced by TGF- $\beta$ or glucosylceramide synthase inhibitor EtDO-P4 (39). The TGF- $\beta$ signaling pathway was activated by FUT3 through fucosylation of Type I TGF- $\beta$ receptors kinase in SLea and SLex-producing cancer cells, leading to EMT and augmentation of their malignant potential $(24,40,41)$. The results of the present study demonstrated that the knockdown of FUT3 by shRNA in Capan-1 cells increased the expression of E-cadherin and decreased the rate of TGF- $\beta$-induced EMT, which was in accordance with previous studies $(7,24)$.

The interaction between E-selectin and SLex may be blocked by their corresponding antagonists. However, the normal function of leukocytes was disrupted inevitably following the introduction of these reagents $(13,37)$. As all E-selectin ligands share the SLex module, we hypothesize that fucosylation is critical for SLex function. Due to the different expression levels of fucosyltransferase in tumors compared with normal tissues, fucosyltransferases may be a more suitable therapeutic target.

In conclusion, the results of the present study suggest that knocking down of FUT3 was sufficient to impair tumorigenesis in vivo. In consideration of its role in cell proliferation and migration, there is potential value in FUT3 as a therapeutic target to develop novel anti-adhesion therapies for pancreatic cancer.

\section{Acknowledgements}

The authors would like to thank Dr Peng Wang from Fudan University Shanghai Cancer Center (Shanghai, China) for his advice.

\section{Funding}

This study was supported by the National Science Foundation of China (grant nos., 81273953 and 81273955).

\section{Availability of data and materials}

The datasets used or analyzed during the present study are available from the corresponding author on reasonable request.

\section{Authors' contributions}

LZ performed all of the experiments. $\mathrm{LZ}$ and LC analyzed data and wrote the manuscript. ZC designed experiments and revised final version of this paper. All authors read and approved the final manuscript.

\section{Ethics approval and consent to participate}

All studies involving animal manipulations were approved by the Fudan University Shanghai Medical College Animal Care and Use Committee (Shanghai, China) and followed the National Institutes of Health guidelines for the care and use of animals (approval no. 20150330A035).

\section{Consent for publication}

Not applicable.

\section{Competing interests}

The authors declare that they have no competing interests.

\section{References}

1. Siegel R, Naishadham D and Jemal A: Cancer statistics, 2012. CA Cancer J Clin 62: 10-29, 2012.

2. Kamisawa T, Wood LD, Itoi T and Takaori K: Pancreatic cancer. Lancet 388: 73-85, 2016.

3. Real FX: A 'catastrophic hypothesis' for pancreas cancer progression. Gastroenterology 124: 1958-1964, 2003.

4. Park JJ, Yi JY, Jin YB, Lee YJ, Lee JS, Lee YS, Ko YG and Lee M: Sialylation of epidermal growth factor receptor regulates receptor activity and chemosensitivity to gefitinib in colon cancer cells. Biochem Pharmacol 83: 849-857, 2012.

5. Padró M, Mejías-Luque R, Cobler L, Garrido M, Pérez-Garay M, Puig S, Peracaula R and de Bolós C: Regulation of glycosyltransferases and Lewis antigens expression by IL-1beta and IL-6 in human gastric cancer cells. Glycoconj J 28: 99-110, 2011.

6. Kannagi R, Izawa M, Koike T, Miyazaki K and Kimura N: Carbohydrate-mediated cell adhesion in cancer metastasis and angiogenesis. Cancer Sci 95: 377-384, 2004.

7. Sakuma K, Aoki M and Kannagi R: Transcription factors c-Myc and CDX2 mediate E-selectin ligand expression in colon cancer cells undergoing EGF/bFGF-induced epithelial-mesenchymal transition. Proc Natl Acad Sci USA 109: 7776-7781, 2012.

8. Marques ETA Jr: Fucosyltransferases In. In 'Carbohydrate in chemistry and biology': Weinheim: Wiley-VCH: 197-211, 2000.

9. Clarke JL and Watkins W: Alpha1,3-L-fucosyltransferase expression in developing human myeloid cells. Antigenic, enzymatic, and mRNA analyses. J Biol Chem 271: 10317-10328, 1996.

10. Desiderio V, Papagerakis P, Tirino V, Zheng L, Matossian M, Prince ME, Paino F, Mele L, Papaccio F, Montella R, et al: Increased fucosylation has a pivotal role in invasive and metastatic properties of head and neck cancer stem cells. Oncotarget 6: 71-84, 2015.

11. Kukowska-Latallo JF, Larsen RD, Nair RP and Lowe JB: A cloned human cDNA determines expression of a mouse stage-specific embryonic antigen and the Lewis blood group alpha(1,3/1,4)fucosyltransferase. Genes Dev 4: 1288-1303, 1990.

12. Ben-David T, Sagi-Assif O, Meshel T, Lifshitz V, Yron I and Witz IP: The involvement of the sLe-a selectin ligand in the extravasation of human colorectal carcinoma cells. Immunol Lett 116: 218-224, 2008

13. Mathieu S, Prorok M, Benoliel AM, Uch R, Langlet C, Bongrand P, Gerolami R and El-Battari A: Transgene expression of alpha(1,2)-fucosyltransferase-I (FUT1) in tumor cells selectively inhibits sialyl-Lewis $\mathrm{x}$ expression and binding to E-selectin without affecting synthesis of sialyl-Lewis a or binding to P-selectin. Am J Pathol 164: 371-383, 2004.

14. Zipin A, Israeli-Amit M, MeshelT, Sagi-AssifO, Yron I, Lifshitz V, Bacharach E, Smorodinsky NI, Many A, Czernilofsky PA, et al: Tumor-microenvironment interactions: The fucose-generating FX enzyme controls adhesive properties of colorectal cancer cells. Cancer Res 64: 6571-6578, 2004.

15. Pérez-Garay M, Arteta B, Llop E, Cobler L, Pagès L, Ortiz R, Ferri MJ, de Bolós C, Figueras J, de Llorens R, et al: $\alpha 2,3$-Sialyltransferase ST3Gal IV promotes migration and metastasis in pancreatic adenocarcinoma cells and tends to be highly expressed in pancreatic adenocarcinoma tissues. Int $\mathrm{J}$ Biochem Cell Biol 45: 1748-1757, 2013. 
16. Aubert M, Panicot-Dubois L, Crotte C, Sbarra V, Lombardo D, Sadoulet MO and Mas E: Peritoneal colonization by human pancreatic cancer cells is inhibited by antisense FUT3 sequence. Int J Cancer 88: 558-565, 2000.

17. Weston BW, Hiller KM, Mayben JP, Manousos GA, Bendt KM, Liu R and Cusack JC Jr: Expression of human alpha(1,3)fucosyltransferase antisense sequences inhibits selectin-mediated adhesion and liver metastasis of colon carcinoma cells. Cancer Res 59: 2127-2135, 1999.

18. Padro M, Cobler L, Garrido M and de Bolos C: Down-regulation of FUT3 and FUT5 by shRNA alters Lewis antigens expression and reduces the adhesion capacities of gastric cancer cells. Biochim Biophys Acta 1810: 1141-1149, 2011.

19. Kalluri R and Weinberg RA: The basics of epithelial-mesenchymal transition. J Clin Invest 119: 1420-1428, 2009.

20. Thiery JP, Acloque H, Huang RY and Nieto MA: Epithelial-mesenchymal transitions in development and disease. Cell 139: 871-890, 2009.

21. Kalluri R: EMT: When epithelial cells decide to become mesenchymal-like cells. J Clin Invest 119: 1417-1419, 2009.

22. Mani SA, Guo W, Liao MJ, Eaton EN, Ayyanan A, Zhou AY, Brooks M, Reinhard F, Zhang CC, Shipitsin M, et al: The epithelial-mesenchymal transition generates cells with properties of stem cells. Cell 133: 704-715, 2008

23. Radisky DC and LaBarge MA: Epithelial-mesenchymal transition and the stem cell phenotype. Cell Stem Cell 2: 511-512, 2008.

24. Hirakawa M, Takimoto R, Tamura F, Yoshida M, Ono M, Murase K, Sato Y, Osuga T, Sato T, Iyama S, et al: Fucosylated TGF- $\beta$ receptors transduces a signal for epithelial-mesenchymal transition in colorectal cancer cells. Br J Cancer 110: 156-163, 2014.

25. Breiman A, López Robles MD, de Carné Trécesson S, Echasserieau K, Bernardeau K, Drickamer K, Imberty A Barillé-Nion S, Altare F and Le Pendu J: Carcinoma-associated fucosylated antigens are markers of the epithelial state and can contribute to cell adhesion through CLEC17A (Prolectin). Oncotarget 7: 14064-14082,2016.

26. National Research Council: Guide for the Care and Use of Laboratory Animals. The National Academies Press, Washington, DC, 1996

27. Dull T, Zufferey R, Kelly M, Mandel RJ, Nguyen M, Trono D and Naldini L: A third-generation lentivirus vector with a conditional packaging system. J Virol 72: 8463-8471, 1998.

28. Bassagañas S, Allende H, Cobler L, Ortiz MR, Llop E, de Bolós C and Peracaula R: Inflammatory cytokines regulate the expression of glycosyltransferases involved in the biosynthesis of tumor-associated sialylated glycans in pancreatic cancer cell lines. Cytokine 75: 197-206, 2015.

29. Radhakrishnan P, Beum PV, Tan S and Cheng PW: Butyrate induces sLex synthesis by stimulation of selective glycosyltransferase genes. Biochem Biophys Res Commun 359: 457-462, 2007.

30. Li Z, Yamada S, Inenaga S, Imamura T, Wu Y, Wang KY, Shimajiri S, Nakano R, Izumi H, Kohno K and Sasaguri Y: Polypeptide $\mathrm{N}$-acetylgalactosaminyltransferase 6 expression in pancreatic cancer is an independent prognostic factor indicating better overall survival. Br J Cancer 104: 1882-1889, 2011.
31. Taniuchi K, Cerny RL, Tanouchi A, Kohno K, Kotani N, Honke K, Saibara T and Hollingsworth MA: Overexpression of GalNAc-transferase GalNAc-T3 promotes pancreatic cancer cell growth. Oncogene 30: 4843-4854, 2011.

32. Iwai K, Ishikura H, Kaji M, Sugiura H, Ishizu A, Takahashi C, Kato $\mathrm{H}$, Tanabe $\mathrm{T}$ and Yoshiki T: Importance of E-selectin (ELAM-1) and sialyl Lewis(a) in the adhesion of pancreatic carcinoma cells to activated endothelium. Int J Cancer 54: 972-977, 1993.

33. Kishimoto T, Ishikura H, Kimura C, Takahashi T, Kato H and Yoshiki T: Phenotypes correlating to metastatic properties of pancreas adenocarcinoma in vivo: The importance of surface sialyl Lewis(a) antigen. Int J Cancer 69: 290-294, 1996.

34. Nakagoe T, Sawai T, Tsuji T, Jibiki MA, Nanashima A, Yamaguchi $\mathrm{H}$, Yasutake T, Ayabe H, Arisawa K and Ishikawa H: Difference in prognostic value between sialyl Lewis(a) and sialyl Lewis(x) antigen levels in the preoperative serum of gastric cancer patients. J Clin Gastroenterol 34: 408-415, 2002.

35. Nakagoe T, Sawai T, Tsuji T, Jibiki M, Ohbatake M, Nanashima A, Yamaguchi H, Kurosaki N, Yasutake T, Ayabe H and Tagawa Y: Difference in prognostic value between sialyl Lewis(a) and sialyl lewis(x) antigens in blood samples obtained from the drainage veins of the colorectal tumors. Cancer Lett 159: 159-168, 2000.

36. Julien S, Ivetic A, Grigoriadis A, QiZe D, Burford B, Sproviero D Picco G, Gillett C, Papp SL, Schaffer L, et al: Selectin ligand sialyl-Lewis $\mathrm{x}$ antigen drives metastasis of hormone-dependent breast cancers. Cancer Res 71: 7683-7693, 2011.

37. Yin X, Rana K, Ponmudi V and King MR: Knockdown of fucosyltransferase III disrupts the adhesion of circulating cancer cells to E-selectin without affecting hematopoietic cell adhesion. Carbohydr Res 345: 2334-2342, 2010

38. Yadav A, Kumar B, Yu JG, Old M, Teknos TN and Kumar P: Tumor-associated endothelial cells promote tumor metastasis by chaperoning circulating tumor cells and protecting them from anoikis. PLoS One 10: e141602, 2015.

39. Guan F, Handa K and Hakomori SI: Specific glycosphingolipids mediate epithelial-to-mesenchymal transition of human and mouse epithelial cell lines. Proc Natl Acad Sci USA 106: 7461-7466, 2009.

40. Fischer KR, Durrans A, Lee S, Sheng J, Li F, Wong ST, Choi H, El Rayes T, Ryu S, Troeger J, et al: Epithelial-to-mesenchymal transition is not required for lung metastasis but contributes to chemoresistance. Nature 527: 472-476, 2015.

41. Zheng X, Carstens JL, Kim J, Scheible M, Kaye J, Sugimoto H, Wu CC, LeBleu VS and Kalluri R: Epithelial-to-mesenchymal transition is dispensable for metastasis but induces chemoresistance in pancreatic cancer. Nature 527: 525-530, 2015

(i) $($ This work is licensed under a Creative Commons Attribution-NonCommercial-NoDerivatives 4.0 International (CC BY-NC-ND 4.0) License. 